\title{
Adiabatic quantum computation and Deutsch's algorithm
}

\author{
Saurya Das, ${ }^{*}$ Randy Kobes, ${ }^{\dagger}$ and Gabor Kunstatter ${ }^{\ddagger}$ \\ Physics Department, The University of Winnipeg and Winnipeg Institute for Theoretical Physics, 515 Portage Avenue, Winnipeg, \\ Manitoba, Canada R3B 2E9
}

(Received 21 November 2001; published 5 June 2002)

\begin{abstract}
We show that by a suitable choice of a time-dependent Hamiltonian, Deutsch's algorithm can be implemented by an adiabatic quantum computer. We extend our analysis to the Deutsch-Jozsa problem and estimate the required running time for both global and local adiabatic evolutions.
\end{abstract}

DOI: 10.1103/PhysRevA.65.062310

PACS number(s): 03.67.Lx

Quantum computation and quantum information theory have attracted a great deal of attention in recent times. Inherently quantum-mechanical systems can in principle be used to implement a wide variety of computational algorithms with enhanced efficiency [1-3]. The principle of superposition in quantum mechanics, according to which a system can be in a linearly superposed state of more than one eigenstate, is the key to this increased efficiency. One of the first algorithms that was first proposed in this context is Deutsch's algorithm [4].

In this, one would like to determine whether a function

$$
f:\{0,1\} \rightarrow\{0,1\}
$$

is constant or balanced, i.e., whether $f(0)=f(1)$ or $f(0)$ $\neq f(1)$ using a quantum computer.

The four possible outcomes of $f$ are

$$
\begin{gathered}
f(0)=f(1)=0 \quad(\text { constant }), \\
f(0)=f(1)=1 \quad(\text { constant }), \\
f(0)=0, f(1)=1 \quad \text { (balanced), } \\
f(0)=1, f(1)=0 \quad \text { (balanced). }
\end{gathered}
$$

Ordinarily, one has to determine both $f(0)$ and $f(1)$ to infer the nature of the function, since the knowledge of one does not shed light on the value of the other. However, it was shown that by applying a certain sequence of unitary operators (gates) on a given initial quantum-mechanical state, and then making just one measurement on the final state, the nature of the function $f$ can be determined [4].

Recently, a new framework of quantum computation has been proposed, in which the series of gates referred to above is entirely replaced by a Hamiltonian that changes continuously with time. The Hamiltonian is so chosen that the state of the system is its ground state at all times (although the ground state itself is time dependent), and the system slowly evolves to a desired final state [5]. Several applications of this have been considered [6]. Using this framework, it was shown that Grover's search algorithm can be efficiently

\footnotetext{
*Electronic address: saurya@ theory.uwinnipeg.ca

†Electronic address: randy@theory.uwinnipeg.ca

*Electronic address: gabor@theory.uwinnipeg.ca
}

implemented [7]. In this paper, we show that Deutsch's algorithm can be implemented as well, by choosing a suitable initial state and a Hamiltonian which evolves that state. Then a single measurement of the final state suffices to determine whether the function $f$ is constant or balanced. Finally, we show that the results can be extended to the Deutsch-Jozsa algorithm involving $n$ qubits.

Let us begin with a two-level system, e.g., a spin $1 / 2$ particle, with the basis kets $\{|0\rangle,|1\rangle\}$. We define the "initial" and "final" Hamiltonians $H_{0}$ and $H_{1}$, respectively, as

$$
\begin{aligned}
& H_{0}=I-\left|\psi_{0}\right\rangle\left\langle\psi_{0}\right|, \\
& H_{1}=I-\left|\psi_{1}\right\rangle\left\langle\psi_{1}\right|,
\end{aligned}
$$

where the initial and final state vectors are given, respectively, by

$$
\begin{gathered}
\left|\psi_{0}\right\rangle=\frac{1}{\sqrt{2}}(|0\rangle+|1\rangle), \\
\left|\psi_{1}\right\rangle=\alpha|0\rangle+\beta|1\rangle,
\end{gathered}
$$

with

$$
\begin{aligned}
& \alpha=\frac{1}{2}\left|(-1)^{f(0)}+(-1)^{f(1)}\right|, \\
& \beta=\frac{1}{2}\left|(-1)^{f(0)}-(-1)^{f(1)}\right| .
\end{aligned}
$$

Note that the Hamiltonians in the above are implicitly given in terms of some fundamental energy scale, $\bar{E}$, say, whose value is determined by the physical system used to construct the states. This energy scale has a natural time scale associated with it, namely, $\bar{T}=\hbar / \bar{E}$, which will play an important role later. The following relations will also prove useful:

$$
\begin{gathered}
\alpha+\beta=1, \\
\alpha^{2}=\alpha, \quad \beta^{2}=\beta,
\end{gathered}
$$

and

$$
\alpha \beta=0
$$


Thus, when $f$ is a constant, $\alpha=1, \beta=0$, and vice versa. We assume that, the system is initially in a state $\left|\psi_{0}\right\rangle$ and is evolved by the following time dependent Hamiltonian:

$$
H(t)=[1-s(t)] H_{0}+s(t) H_{1} .
$$

In general, $s(t)$ is an arbitrary function of the time $t$, such that $s(0)=0$ and $s(1)=1$. Thus, $H(0)=H_{0}$ and $H(1)$ $=H_{1}$. For the present we assume that $s(t)$ is linear in $t$, namely, $s=t / T$, where $T$ is the total time for which the system is evolved.

It follows from the adiabatic theorem that at $t=T$, the system would be in the state $\left|\psi_{1}\right\rangle$, with very high probability $1-\epsilon^{2}$ (where $\epsilon$ is a small number), provided the evolution is adiabatic $[8,9]$, i.e.,

$$
\frac{\left|\left\langle\frac{d H}{d t}\right\rangle\right|}{g_{\min }^{2}} \leqslant \epsilon
$$

where the time $t$ is implicitly given in units of $\bar{T}$. The lower bound on the evolution time $T$ is implicitly given by the condition (11) as will be seen later and

$$
\left\langle\frac{d H}{d s}\right\rangle:=\left\langle E_{+}, s\left|\frac{d H}{d t}\right| E_{-}, s\right\rangle
$$

and

$$
\left\langle\frac{d H}{d t}\right\rangle=\frac{d s}{d t}\left\langle\frac{d H}{d s}\right\rangle .
$$

$E_{+}(s)$ and $E_{-}(s)$ being the two time dependent eigenvalues of $H(s)$, with corresponding eigenvectors $\left|E_{+}, s\right\rangle$ and $\left|E_{-}, s\right\rangle$, respectively, and

$$
g_{\min }:=\min _{0 \leqslant s \leqslant 1}\left[E_{+}(s)-E_{-}(s)\right] .
$$

Of course, the quantities $\langle d H / d t\rangle$ and $g_{\min }$ should be nonvanishing. We will show that this is indeed the case.

Thus, under adiabatic quantum evolution, the initial wave function of the system will evolve to either $|0\rangle$ or $|1\rangle$, almost with certainty, and by making a single measurement of the state at the end, one can determine unambiguously what the nature of the function $f$ is. Namely, if the outcome of measurement is $|0\rangle$, then $f$ is constant and if it is $|1\rangle$, then it is balanced.

The matrix elements of $H$ in the $\{|0\rangle,|1\rangle\}$ basis are

$$
H(s)=\left(\begin{array}{cc}
1 / 2+s(\beta-1 / 2) & -1 / 2(1-s) \\
-1 / 2(1-s) & 1 / 2+s(\alpha-1 / 2)
\end{array}\right) .
$$

The corresponding eigenvalues are

$$
E_{ \pm}(s)=\frac{1}{2}\left[1 \pm \sqrt{1-2 s+2 s^{2}}\right] .
$$

Note that the eigenvalues are independent of $\alpha$ and $\beta$, and are identical to those found in [7]. This is a consequence of the Eqs. (7)-(9). It follows that

$$
\Delta E(s)=E_{+}(s)-E_{-}(s)=\sqrt{1-2 s+2 s^{2}},
$$

which is nonzero for all values of $s$ and

$$
g_{\min }=\Delta E(s=1 / 2)=\frac{1}{\sqrt{2}} .
$$

In addition, the two orthonormal eigenvectors are

$$
\left|E_{ \pm}, s\right\rangle=k_{ \pm}\left(\begin{array}{c}
1-s \\
{\left[(1-2 \alpha) s \mp \sqrt{1-2 s+2 s^{2}}\right]}
\end{array}\right),
$$

where

$$
k_{ \pm}=2^{-1 / 2}\left\{\left(1-2 s+2 s^{2}\right) \pm(2 \alpha-1) s \sqrt{1-2 s+2 s^{2}}\right\}^{-1 / 2}
$$

with which one can get

$$
\left|\left\langle\frac{d H}{d t}\right\rangle\right|=\frac{1}{T} \frac{1}{2 \sqrt{1-2 s+2 s^{2}}} .
$$

Note that this too is independent of $\alpha$ and $\beta$ although $\left|E_{ \pm}, s\right\rangle$ is not. Also, note that for any value of $s$, the above quantity is nonvanishing and of order $1 / T$. At the final time $t=T$, the eigenstates are $|0\rangle$ and $|1\rangle$, respectively, and depending on the value of $\alpha, \beta$, the system evolves to one of them.

Substituting Eqs. (15) and (16) in Eq. (11), we get the following relation:

$$
T \geqslant \frac{1}{\epsilon}
$$

which gives an estimate of the time for which the initial state $\left|\psi_{0}\right\rangle$ must be evolved via the adiabatic Hamiltonian (10) to attain an accuracy of order $\epsilon$ of the final result. For example, if we want the final state to be the $\left|\psi_{1}\right\rangle$ with accuracy of $90 \%$, then the minimum evolution time should be on the order of $T \approx 1 / \sqrt{1-0.9} \approx 3.2$, in units of $\bar{T}$.

A few comments are in order here. Instead of starting with the initial state (0.4), one can in general start with an arbitrary initial state of the form

$$
\left|\psi_{0}\right\rangle=a|0\rangle+b|1\rangle
$$

with $|a|^{2}+|b|^{2}=1$, and evolve the system with the Hamiltonian (10). The end result is expected to remain unchanged, since the ground state of the final Hamiltonian is still $\left|\psi_{1}\right\rangle$, to which the system will eventually tend. If one starts with the state (18), then $\Delta(s)$ and $\langle d H / d t\rangle$ are, respectively,

$$
\begin{gathered}
\Delta(s)=\sqrt{1-4\left(a^{2} \beta+b^{2} \alpha\right) s(1-s)} \\
\left|\left\langle\frac{d H}{d t}\right\rangle\right|=\frac{1}{T} \frac{a b}{\sqrt{1-4\left(a^{2} \beta+b^{2} \alpha\right) s(1-s)}} .
\end{gathered}
$$

Substituting Eqs. (19), (20), and $s=1 / 2$ in Eq. (11) and simplifying we get 


$$
T \geqslant \frac{1}{\epsilon} \frac{a b}{\sqrt{b^{2}\left(1-a^{2}\right)+\alpha\left(a^{2}-b^{2}\right)}} .
$$

Thus depending on whether $\alpha=0$ or $\alpha=1$ (although this value is a priori unknown), we get, respectively,

$$
T \geqslant \frac{1}{\epsilon} \frac{a}{b} \quad \text { or } \quad T \geqslant \frac{1}{\epsilon} \frac{b}{a} .
$$

Thus for extreme asymmetric values of $a$ and $b$ (e.g., $a \approx 0$ and $b \approx 1$ ), the evolution into the final state would either take place in a very short or a very long time. But as the value of $\alpha$ is not known, one would have to wait for the greater of two values before making the measurement. Equivalently, for very small $a$ or $b, g_{\text {min }}$ becomes very small for some value of $\alpha$, which is contrary to what the validity of adiabatic theorem requires. Consequently, the "optimal" values for which the evolution time is independent of $\alpha, \beta$ is given by

$$
a=b=\frac{1}{\sqrt{2}},
$$

which is what we started with.

The above procedure can be generalized to Boolean functions of the form

$$
f:\{0,1\}^{n} \rightarrow\{0,1\}
$$

by making use of $n$ qubits instead of a single one $[1,10]$. In accordance with the Deutsch-Jozsa problem, we assume that it is "promised" that the function is either constant (i.e., all outputs are identical) or balanced (i.e., has an equal number of 0's and 1's as outputs), and the task is to find which of the above it actually is $[1,10]$. The basis states now are $\{|0\rangle,|1\rangle, \ldots,|N-1\rangle\}$ (with $N=2^{n}$ ). Now we choose the normalized initial and final states to be

$$
\begin{gathered}
\left|\psi_{0}\right\rangle=\frac{1}{\sqrt{N}} \sum_{i=0}^{N-1}|i\rangle, \\
\left|\psi_{1}\right\rangle=\alpha|0\rangle+\frac{\beta}{\sqrt{N-1}} \sum_{k=1}^{N-1}|k\rangle,
\end{gathered}
$$

with

$$
\begin{gathered}
\alpha=\frac{1}{N}\left|\sum_{x \in\{0,1\}^{n}}(-1)^{f(x)}\right|, \\
\beta=1-\alpha .
\end{gathered}
$$

Once again, if $f(x)$ is constant then $\alpha=1$ and $\beta=0$, and vice versa. Thus after the required running time, if a measurement of the final state yields $|0\rangle$, then $f(x)$ is constant and if it does not yield $|0\rangle$, then it is balanced. The properties (7)-(9) continue to be valid. Also, $H_{0}, H_{1}$, and $H(s)$ are still given by Eqs. (1), (2), and (10), respectively.

In the chosen basis, the adiabatic Hamiltonian $H(s)$ is now given by the following $N \times N$ matrix:

$$
H(s)=\left(\begin{array}{ccccc}
1-\frac{1-s}{N}-\alpha s & -\frac{1-s}{N} & -\frac{1-s}{N} & \cdots & -\frac{1-s}{N} \\
-\frac{1-s}{N} & 1-\frac{1-s}{N}-\frac{s \beta}{N-1} & -\frac{1-s}{N}-\frac{s \beta}{N-1} & \cdots & -\frac{1-s}{N}-\frac{s \beta}{N-1} \\
-\frac{1-s}{N} & -\frac{1-s}{N}-\frac{s \beta}{N-1} & 1-\frac{1-s}{N}-\frac{s \beta}{N-1} & \cdots & -\frac{1-s}{N}-\frac{s \beta}{N-1} \\
\cdots & \cdots & \cdots & \cdots & \cdots \\
-\frac{1-s}{N} & -\frac{1-s}{N}-\frac{s \beta}{N-1} & -\frac{1-s}{N}-\frac{s \beta}{N-1} & \cdots & 1-\frac{1-s}{N}-\frac{s \beta}{N-1}
\end{array}\right)
$$

It can be shown that the highest eigenvalue of the above Hamiltonian is 1 , which is $(N-2)$-fold degenerate, and the two remaining distinct eigenvalues (both less than 1) are

$$
E_{ \pm}(s)=\frac{1}{2}\left[1 \pm \sqrt{1-\frac{4 s(1-s)}{N}[\beta+\alpha(N-1)]}\right] .
$$

Thus,

$$
\begin{aligned}
\Delta E(s) & =E_{+}(s)-E_{-}(s)=\sqrt{1-\frac{4 s(1-s)}{N}[\beta+\alpha(N-1)]} \\
& \neq 0
\end{aligned}
$$

implying

$$
g_{\min }=\Delta E(s=1 / 2)=\sqrt{1-\frac{1}{N}[\beta+\alpha(N-1)]} .
$$




$$
\left|\left\langle\frac{d H}{d s}\right\rangle\right|=\frac{\sqrt{N-1}}{N} \frac{1}{\sqrt{1-\frac{4 s(1-s)}{N}[\beta+\alpha(N-1)]}} .
$$

Thus for $s=t / T$, from condition (11) it follows that (for large $N$ )

$$
T \geqslant \frac{N}{\epsilon},
$$

which shows that the evolution time scales as $N$, the number of qubits.

However, following Ref. [7] if we assume an evolution with a general $s(t)$ we obtain the adiabaticity condition (11) that must be satisfied at any given instant of time $t$,

$$
\frac{d s}{d t} \leqslant \epsilon \frac{\left[E_{+}(s)-E_{-}(s)\right]^{2}}{\left|\left\langle\frac{d H}{d s}\right\rangle\right|} .
$$

Substituting Eqs. (28) and (30), we get (here $\gamma \equiv 4[\beta$ $+\alpha(N-1)] / N)$,

$$
\int_{0}^{T} d t \geqslant \frac{1}{\epsilon} \frac{\sqrt{N-1}}{N} \int_{0}^{1} \frac{d s}{\left[1-s \gamma+s^{2} \gamma\right]^{3 / 2}}
$$

The expression on the right-hand side can be integrated, and is equal to

$$
\frac{1}{\epsilon}\left[\frac{\sqrt{N-1}}{N} \frac{1}{\gamma^{3 / 2}} \frac{2 s-1}{\left(\gamma^{-1}-1 / 4\right) \sqrt{s^{2}-s+\gamma^{-1}}}\right] .
$$

Inserting the limits of $s$, we get the following bound:

$$
T \geqslant \frac{1}{\epsilon} \frac{\sqrt{N-1}}{N} \frac{1}{\gamma(1-\gamma / 4)} .
$$

It can be verified that for $N \gg 1$, this lower bound is

$$
T \geqslant \frac{\sqrt{N}}{\epsilon},
$$

which is a quadratic improvement over the previous bound (31). Also since relation (32) has to be satisfied at every instant, the bound (35) is optimal.

To conclude, in this paper, we have implemented Deutsch's algorithm using adiabatic quantum evolution by a Hamiltonian that takes a given initial state to a final state such that the final state depends on the nature of the function $f$. In particular, if the function is constant, the final state is $|0\rangle$ with a very high probability, and if it is balanced then the outcome is $|1\rangle$ almost with certainty. Then a measurement on the final state helps to determine the nature of $f$. We have also estimated the required evolution time for a given accuracy of the result. Finally, we have generalized the result for the Deutsch-Jozsa problem, using $n$ qubits, and found that the number of time steps required to solve the problem scales as $\sqrt{N}$, where $N=2^{n}$. Although this is a marginal improvement over the classically required exponential time ( of order $N$ ), it does not match the polynomial time that is achievable using standard quantum computational techniques [10]. It would be interesting to compare adiabatic and standard quantum computational methods for other algorithms to see whether this difference in computational time is the exception rather than the rule. We hope to report on this elsewhere.

We would like to thank J. Currie, R. Laflamme, and V. Linek for useful discussions. This work was supported in part by the Natural Sciences and Engineering Research Council of Canada.
[1] R. Cleve, A. Ekert, C. Macchiavello, and M. Mosca, Proc. R. Soc. London, Ser. A 454, 339 (1998).

[2] E. Rieffel and W. Polak, e-print quant-ph/9809016.

[3] A. Ekert, P. Hayden, and H. Inamori, e-print quant-ph/0011013.

[4] D. Deutsch, Proc. R. Soc. London, Ser. A 400, 97 (1985).

[5] E. Farhi, J. Goldstone, S. Gutmann, and M. Sipser, e-print quant-ph/0001106; A. M. Childs, E. Farhi, and J. Preskill, e-print quant-ph/0108048; E. Farhi and S. Gutmann, e-print quant-ph/9612026.

[6] E. Farhi, J. Goldstone, S. Gutmann, J. Lapan, A. Lundgren, and D. Preda, e-print quant-ph/0104129; A. M. Childs, E. Farhi, J. Goldstone, and S. Gutmann, e-print quant-ph/0012104; E. Farhi, J. Goldstone, and S. Gutmann, e-print quant-ph/0007071.

[7] J. Roland and J. Cerf, e-print quant-ph/0107015.

[8] A. Messiah, Quantum Mechanics (North-Holland, Amsterdam, 1976), Vol. II.

[9] B. H. Bransden and C. J. Joachain, Introduction to Quantum Mechanics (Harlow, Essex, England, 1989).

[10] D. Deutsch and R. Jozsa, Proc. R. Soc. London, Ser. A 439, 553 (1992). 